\title{
Session 5: Molecular Biology I
}

[09.30-09.30]

'Understanding the biology of human IgG4'

Shirley Peters

UCB-New Medicines, Slough, UK

Abstract not provided.

[09.30-09.50]

'Rapid discovery and optimization of therapeutic human antibodies using Adimabs yeast based discovery platform'

Jonathan Belk

Adimab Inc., Lebanon, NH, USA

We have created a synthetic antibody library with diversity that closely mimics the key features of the pre-immune process of VDJ recombination. This library has been synthesized and introduced into a novel yeast based expression system. This highly integrated platform provides access to significant amounts of high quality, full-length IgG protein within one month of the initiation of the selection process. The ability to assay the bioactivity and biophysical properties of hundreds of therapeutic leads in such a brief time period has lead to a shift in the traditional paradigm of biologics discovery, ultimately reducing the time and cost to identify and develop antibody therapeutics.
[09.50-10.10]

'Effect of IgG subclass or allotype on the ability to generate a high expressing mammalian cell line and high yielding antibody manufacturing process' Gregory T. Bleck

Catalent Pharma Solutions, Middleton, WI, USA

Cell lines producing IgG1 (multiple different allotypes), IgG2, IgG4 (native sequence) and IgG4 (hinge mutation (serine 241 to proline)) antibodies were generated by GPEx ${ }^{\circledR}$ and used for clinical manufacturing. GPEx ${ }^{\circledR}$ is a versatile protein expression system which is capable of transferring genes of interest into a wide variety of mammalian host cells and offers a number of advantages over other methods for production of antibodies. These advantages include shorter timelines, improved consistency, higher specific productivity, and better genetic stability. Cell lines and processes yielding between 2 and $5 \mathrm{~g} / \mathrm{L}$ were developed for each of the different antibody allotypes and subclasses. Specific productivities for each of cell lines ranged between 30 and 90 picograms/cell/day. Data to date indicate that neither the type of IgG allotype or subclass appear to directly impact the ability to obtain a highly productive cell line and efficient manufacturing process. Our experience making $\mathrm{CHO}$ cell lines producing over 200 different IgG's will be summarized, and case studies of antibody cell line generation and manufacturing process development will be discussed. 